\title{
Psychological aspects of cancer
}

\author{
S.S. Ratnatunga ${ }^{1}$, K.C. Ratnatunga ${ }^{2}$, V.A. De Silva ${ }^{3}$ \\ 1 University Psychiatry Unit, National Hospital of Sri Lanka \\ 2 University Surgical Unit, Colombo South Teaching Hospital, Sri Lanka \\ 3 Department of Psychiatry, Faculty of Medicine, University of Colombo, Sri Lanka
}

\section{Introduction}

Cancer as a disease entity is perhaps one of the most physically and psychologically destructive diseases. Its' relentlessness, the aggression of treatment, and perceived unconquerability contribute to significant physical and psychological morbidity. The role of psychiatry and psychology in the holistic management of these patients cannot be overemphasized; but is unfortunately, often understated.

In this review we attempt to profile the scope of the sciences of the mind within the larger framework of oncology, from the psychological impact of the diagnosis, recurrence and metastasis, to psychological reaction in relation to cancer treatment. We also touch upon the methods of psychological treatment, communication with cancer patients and their families, and dealing with terminal care.

In the United States of America, Memorial Sloan Kettering Cancer Center (MSK) pioneered the introduction of psychiatry in the oncology setting. The service which started in the 1970s offers diagnosis and management of psychiatric disorders, neuropsychological evaluation, psychotherapy services to the patient and family, and care giver and bereavement support [1]. The wide range of services offered at this institute reflects the importance placed on helping patients and families deal with the emotional consequences of their illness.

The diagnosis of cancer evokes a far greater emotional response than perhaps any other disease. The natural response of shock, disbelief and denial are common initial responses, followed by anger, anxiety, depression and the feeling of loss. Typically the grief reaction which occurs as a response to death of a loved one or in anticipation of one's own death is described in five phases: denial, anger, bargaining, depression and acceptance. The reaction shows great individual variation. The intensity, duration and associated symptoms tend to determine whether the emotional reaction is normal or abnormal.

Correspondence: S.S. Ratnatunga
E-mail: suhashini_sri@yahoo.com
DOI: http://10.4038/sljs.v34i2.8264

The Sri Lanka Journal of Surgery 2016; 34(2): 23-25
The emotional reaction to cancer can be described as an existential crisis where a person questions the very foundations of his or her life and wonders if it has any value or purpose. Confrontation with death often evokes anxiety and sadness but for some it can be an opportunity for growth and change. Therapy can help people deal with the anxieties, prioritize what needs to be done and make the best use of their time left.

When people are unable to come to terms with impending death, maladaptive psychological states can occur. Adjustment disorder is the commonest psychiatric diagnosis in patients with cancer [6]. This indicates that psychological symptoms interfere with functioning, treatment decisions and well-being [2].

\section{Depression}

Cancer is associated with higher rates of depression than the general population (20-50\%) [3]. Depression is particularly associated with pancreatic $(33 \%-50 \%)$, oropharyngeal (22$57 \%)$, breast $(1.5-46 \%)$ and lung $(11-44 \%)$ cancers [4]. Depression is also thought to be a common harbinger of subclinical pancreatic cancer.

\section{Suicide}

The suicide rate in cancer patients is double that of the general population. Formal assessment of suicide risk should be carried out in high risk patients such as elderly males (patients aged 65 years or older have higher suicide rates than those younger than 65 years with rates highest amongst men 80 years or older), ongoing or past history of depression, alcohol dependence and poor social support [5]. Prostate, lung, pancreatic, head and neck cancers are particularly associated with high suicide rates. The first year following diagnosis carries a higher risk for completed suicide. Thoughts of suicide, plans and access to means should be assessed routinely.

\section{Multiple somatic complaints}

Multiple somatic complaints can occur through the entire span of the illness. They are commonly observed in patients in remission or in those who have residual disease. Common symptoms are fatigue, pain and abnormal sensations; often 
misinterpreted by patients as signs of disease recurrence, relapse or metastasis. The presence of these symptoms can cause confusion and lead to unnecessary investigations, over treatment or treatment delay. The pathophysiology of somatization in cancer is poorly understood-exaggeration of somatic symptoms, presence of depression and anxiety, and, abnormal illness behaviour are possible explanations. The presence of somatization in cancer has major clinical implications. Firstly, the clinical challenge is in deciding if the somatic symptoms are due to side effects of treatment (chemotherapy, radiotherapy or surgery), a comorbid psychiatric disorder or illness behaviour. Secondly, they interfere with the well-being of the patient with a significant impact on the quality of life. New evidence is emerging that somatization in disease free cancer patients responds well to selective serotonin reuptake inhibitors (SSRI) and serotonin norepinephrine reuptake inhibitors (SNRI) [6].

\section{Psychological aspects of management}

Psychological aspects of palliative care include detection and management of psychological problems, multimodal pain relief, addressing myths regarding opiate addiction, attention to quality of life, end of life decisions, spiritual concerns and care of the terminal stages whilst addressing issues of dying and bereavement in the family [6].

A challenging and significant part of the management is talking about dying with the patient and the family. "How long has he got?", "Is there anything more that can be done?" are questions where the physician often finds himself burdened by the duty to be honest, but also empathetic and humane. Another particular challenge is when the terminally ill person expresses desire for death or physician assisted dying.

\section{Screening for distress, anxiety and depression}

The International Psycho-Oncology Society have set a standard of care involving monitoring distress as the '6th vital sign' [7]. Countries such as Australia and the United States have clearly defined clinical practice pathways for the screening and treatment of anxiety and depression [8]. The Australian Guidelines recommend using brief validated screening tools to screen for distress, depression and anxiety in all patients with cancer [9]. The Edmonton Symptom Assessment Scale is a ten item visual analog scale which can be rated by the patient [10]. It is a good measure of physical symptom distress which has a direct impact on the well-being and quality of life. The National Comprehensive Cancer Network Distress Thermometer For Patients identifies areas for providing supportive services [11]. Supportive services may be provided by support groups, social workers, religious institutes or counsellors. The Hospital Anxiety and Depression Scale can be used to assess the presence of anxiety and depression [12]. These paper and pencil tests can be selfadministered but some patients may need help in completing these tests.

Screening tests tend to overestimate the disease burden. Therefore patients identified by the screening tests should be clinically evaluated. The biological mechanisms of cancer may give rise to symptoms of anorexia, fatigue, and weight loss, which are common symptoms also in depression. Hence the diagnosis of depression should be based not on these, but on psychological symptoms such as anhedonia (inability to experience joy), social withdrawal, depressive cognitions and suicidal ideation. Feelings of worthlessness and guilt can help differentiate between normal sadness and depression. In a busy clinical setting, use of screening tests enables the limited staff to evaluate a large number of patients. The above mentioned screening tests can be translated and adapted for use in our country or we can develop more culture specific tests.

\section{Interventions}

Patients who need psychosocial help identified through screening and clinical assessment can be broadly divided into two groups. Those who need predominantly physical help and those who need predominantly psychosocial help. Those with poor symptom control or experience side effects of medication can be further evaluated and provided physical help.

Most guidelines recommend stepped care with the least intensive intervention delivered first. If this is not adequate more intensive interventions can be offered. Whilst input from the treating physician is essential, support from a social worker, trained nurse or other health worker is also be needed.

\section{Initial management}

The initial interventions consist of patient education and brief emotional support [9]. The anxiety associated with receiving the diagnosis and associated treatment can be allayed by education and clarifying doubts. There is evidence that patients also benefit from relaxation and stress management training. Although these are simple interventions we often do not provide this care for patients. Having a specially trained member of staff is one way of ensuring that these simple interventions can be implemented. In developed countries, peer groups also play a role in providing support.

\section{Role of the multidisciplinary team}

Ideally psychosocial issues in patients with cancer should be managed with the help of a multidisciplinary team. The team should include the surgeon, oncologist and other clinicians managing the physical aspects as well as a psychiatrist and a social worker. Long term management plans should include psychosocial management. The role of the social worker is still not appreciated by the health care system in Sri Lanka. However, in patients with cancer and other chronic 
debilitating illness, social workers can offer much support to improve quality of life.

\section{Further management}

More intensive interventions are needed if patients do not benefit from the above interventions. Referral to a psychiatrist is warranted if there is moderate to severe depression, anxiety or high suicidal risk. Medication and cognitive behaviour therapy may be required in such patients. Side effects and drug interactions must be considered when prescribing antidepressants. Selective serotonin reuptake inhibitors (SSRI) and serotonin noradrenaline reuptake inhibitors (SNRI) are commonly used antidepressants. SSRIs are also effective in treating anxiety. Benzodiazepines are not recommended due to risk of tolerance and dependence. In the elderly this can cause confusion and falls.

Psychiatric morbidity is also associated with cancer treatment. It can greatly influence general well-being and treatment adherence; prolongation of life is at times at the cost of quality of life. Anticipatory nausea and vomiting is seen in $25-30 \%$ of patients who undergo chemotherapy [13]. This response is as a result of classical conditioning where patients develop symptoms even days prior to chemotherapy, to hospital staff, smell of hospitals or even at the sight of the hospital. The best method to avoid development of this troublesome symptom is to adequately prevent both vomiting and nausea at the first exposure to chemotherapy. If anticipatory side effects do develop, behavioural treatment techniques such as systematic desensitization, have shown to be effective. Low dose alprazolam in combination with behavioural techniques have also been shown useful [13].

While advances are made in the field of oncology ranging from early diagnosis to better outcomes by improving quality of care, the parallel advances in improving quality of life in patients with cancer appear to be lagging behind. We need to focus on aspects such as helping cancer patients and their family members in making informed choices in areas such as curative surgery vs palliative care, reconstructive options, specialized colostomy care, disproving myths about radiation and chemotherapy, better management of nausea and vomiting, attention to cosmesis such as alopecia, skin discoloration, effective pain management, financial support and involvement of spiritual personnel [6].
All authors disclose no conflict of interest. Ethics clearance was not obtained as we only conducted a literature review.

\section{References}

1. https://www.mskcc.org/departments/psychiatry-behavioralsciences. (Accessed on 13.03.2016)

2. World Health Organization The ICD-10 Classification of Mental and Behavioural Disorders. Geneva; World Health Organization, 1992

3. Massimo P, Massimo B Depression in cancer patients: a critical review Clin Pract Epidemiol Ment Health 2007; 3:2

4. Massie MJ Prevalence of depression in patients with cancer. J Natl Cancer Inst Monogr 2004; (32): 57-71 http://dx.doi.org/10.1093/jncimonographs/lgh014

5. Anguiano L, Mayer DK, Piven ML, Rosenstein D. A literature review of suicide in cancer patients. Cancer Nurs 2012; 35(4): E14-26 http://dx.doi.org/10.1097/NCC.0b013e31822fc76c

6. Chaturvedi SK Psychiatric oncology: Cancer in mind. Indian J Psychiatry 2012; 54(2): 111-118 http://dx.doi.org/10.4103/0019-5545.99529

7. Holland JC, Bultz BD, National comprehensive Cancer N. The NCCN guideline for distress management: a case for making distress the sixth vital sign. J Natl Compr Canc Netw 2007; 5(1): 3-7.

8. Wagner LI, Spiegel D, Pearman T. Using the science of psychosocial care to implement the new american college of surgeons commission on cancer distress screening standard. $J$ Natl Compr Canc Netw 2013; 11(2):214-21.

9. Butow P, Price MA, Shaw JM, Turner J, Clayton JM, Grimison P, et al. Clinical pathway for the screening, assessment and management of anxiety and depression in adult cancer patients: Australian guidelines. Psycho-oncology. 2015; 24(9): 987-1001. http://dx.doi.org/10.1002/pon.3920

10. Richardson LA, Jones GW. A review of the reliability and validity of the Edmonton Symptom Assessment System. Curr Oncol $2009 ; 16(1): 55$.

11.National Comprehensive Cancer Network Distress Thermometer. Available from:

http://www.nccn.org/patients/resources/life_with_cancer/pdf/n ccn_distress_thermometer.pdf

12. Zigmond AS, Snaith RP. The hospital anxiety and depression scale. Acta Psychiatr Scand 1983; 67(6): 361-70. http://dx.doi.org/10.1111/j.1600-0447.1983.tb09716.x

13. Joseph AR, Morrow GR, Matti SA Anticipatory Nausea and Vomiting. Support Care Cancer 2011; 19(10): 1533-38. http://dx.doi.org/10.1007/s00520-010-0980-0 